\title{
Introduction
}

\section{Carlos Prieto}

\section{(2) OpenEdition}

\section{Journals}

\section{Édition électronique}

URL : http://journals.openedition.org/travailemploi/4187

DOI : 10.4000/travailemploi.4187

ISSN : 1775-416X

\section{Éditeur}

DARES - Ministère du Travail

\section{Édition imprimée}

Date de publication : 15 septembre 2008

Pagination : 7-11

ISSN : 0224-4365

\section{Référence électronique}

Carlos Prieto, «Introduction », Travail et Emploi [En ligne], 115 | juillet-septembre 2008, mis en ligne le 04 mars 2011, consulté le 22 septembre 2020. URL : http://journals.openedition.org/travailemploi/ 4187 ; DOI : https://doi.org/10.4000/travailemploi.4187 


\title{
Introduction
}

\author{
Carlos Prieto $(*)$
}

Rien ne permettait de prévoir, au début des années 1990, que le niveau de l'emploi d'un pays à économie avancée comme l'Espagne pouvait être multiplié par deux en une période de seulement treize ans. Aucun manuel d'économie du travail n'envisage une telle probabilité en dehors du cas de pays en voie de développement. C'est pourtant ce qui s'est produit en Espagne. De 1994 à 2007, la population active espagnole occupée en Espagne est passée de 12,5 millions à 20,3 millions, et a ramené parallèlement le taux de chômage de 24,5\% en 1994 à 8,6\% en 2007, soit une diminution de plus de $62,4 \%$. Une telle augmentation de la population active employée d'un pays, surprenante en elle-même, l'est plus encore si l'on tient compte de deux autres facteurs. Elle s'est produite sur une durée de treize ans, alors que le nombre de personnes employées en 1994 (12,5 millions) n'avait pratiquement pas changé depuis 1976, c'est-à-dire en vingt ans - et, s’il y a eu des changements, ça a toujours été dans le sens d'une diminution de l'emploi (ce point est traité dans l'article de Luis Toharia sur le marché du travail en Espagne de 1976 à 2006). Ensuite, comparativement à d'autres pays européens, il s'agit d'une augmentation substantielle. Ainsi, la croissance de l'emploi dans l'ensemble des pays de l'Union européenne à quinze entre 1994 et 2005 a été de 12,7\% (données Eurostat), pour 41,9\% en Espagne sur la même période (et de 62,4\% deux ans plus tard, soit une augmentation de 30\%). Le taux global d'emploi montre une évolution non moins surprenante: il a augmenté entre 1994 et 2006 de 6\% dans l’Union européenne à quinze, et de 18,4\% en Espagne, soit trois fois plus (comme le montre le graphique 4 dans l'article de Luis Toharia).

Ces données atypiques font de l’emploi espagnol un phénomène social, économique et même politique particulier, qui appelle une analyse approfondie. Pour y parvenir, ce numéro thématique de Travail et Emploi mobilise des économistes et des sociologues espagnols. Croissance forte de l'emploi et diminution importante du chômage apparaissent comme des développements positifs pour la société espagnole, mais ce numéro met en relief, derrière la face «claire » d'un tel phénomène, une face «obscure » qui s’est développée parallèlement.

La forte croissance de l'emploi en Espagne de ces dernières années doit être resituée dans le contexte de son histoire récente. C'est le sujet développé par Luis Toharia dans la première partie de son article. Cette «forte croissance de l'emploi » n'est pas en elle-même un phénomène étrange dans cette histoire. En prenant comme point de départ l'année 1976, «date remarquable du point de vue aussi bien économique que social et politique (et même statistique)» - c'est l'année du début de la transition vers un régime démocratique - on observe qu'il y a eu récemment une autre période de forte croissance: celle qui va de 1985 à 1991, pendant laquelle l'emploi est passé de 11 à 13 millions. Cette intense création d'emplois s'inscrit entre deux périodes à «forte» destruction d'emploi. Le premier trait - ou du moins le plus visible - de la dynamique de l'emploi en Espagne est son évolution pour ainsi dire spasmodique: il n'y a pas de croissance douce ni de diminution douce de l'emploi, mais toujours des croissances ou des réductions très intenses. La différence entre la croissance des dernières années et celle de la deuxième moitié des années quatre-vingt est sa longue durée: la croissance actuelle a connu - on peut parler au passé puisque les chiffres connus pour 2008 annoncent une nouvelle chute que nous évoquerons plus loin - une durée de treize ans, ce qui en termes d'évolution de l'emploi peut être considéré comme une très longue durée, alors que la précédente n’avait duré que six ans.

La création de plus de douze millions d'emplois à partir de 1994 devient compréhensible quand on considère les secteurs économiques concernés et la qualité des emplois créés. L'explication de la croissance de l'emploi en elle-même est l'objet central de l'article d'Immaculada Cebrián. Les travaux des chercheurs espagnols convergent sur un point: l'augmentation de l'emploi est principalement due à l'évolution de l'emploi dans le secteur de la construction, secteur qui est devenu au cours de ces années le principal moteur de l'économie espagnole. Luis Toharia écrit explicitement: «On peut affirmer, sans peur de

(*) Université Complutense de Madrid. Je remercie A. Serrano pour sa lecture de ce texte et les suggestions qu'elle m’a faites. 
se tromper, que le bâtiment a été le secteur moteur de l'économie espagnole ». Immaculada Cebrián dit de son côté: «Le secteur de la construction, qui a toujours affiché une tendance nettement cyclique, connaît depuis la dernière crise - celle de 1992-1994 - une reprise durable. À cette époque, et notamment en 1993, ce secteur a connu une fois de plus des pertes considérables au niveau de l'emploi, mais a su rapidement recréer de nouveaux emplois à partir de 1994. Leur chiffre a aujourd'hui plus que doublé et a atteint un niveau jamais égalé pour toute la période étudiée (il est plus élevé d’environ 130\% que celui enregistré en 1995). Sur la totalité de la production et de l'emploi, il est passé respectivement de 7 à $11 \%$ et de 9 à $13 \%$ ». L'auteure ajoute: "Cette augmentation significative de la part du secteur de la construction dans le PIB - et dans l'emploi - est caractéristique de la seule Espagne». Le rôle de moteur de l'économie et de l'emploi qu'a joué le secteur de la construction ressort du tableau 1 ci-dessous (données actualisées), à travers l'évolution de l'emploi dans une branche d'activité directement connectée à celle de la construction, celle des «activités immobilières et de loyer services aux entreprises ». Dans cette branche, l'augmentation de l'emploi est même plus élevée que dans la construction: 220,5\% de croissance, face à $141,1 \%$ dans la construction. L'augmentation de l'emploi dans ces deux branches, très au-dessus de la moyenne, prend une signification toute particulière puisque les deux additionnées expliquent 36,3\% de l'augmentation totale (alors qu'elles ne représentent que $23 \%$ de la population active occupée totale). Trois autres branches, suivant une dynamique de croissance spécifique, ont augmenté aussi de façon très supérieure à la moyenne: l'hôtellerie (95,7\%), liée au développement important du secteur du tourisme, et les services sociaux, les services à la personne $(97,3 \%)$ et l'emploi domestique $(132,1 \%)$, rattachés, pour les premiers, à des évolutions dans la demande de services de proximité pour les personnes âgées et, pour l'emploi domestique, à l'entrée massive des femmes dans l'emploi. Il faut signaler aussi la croissance particulière de l'emploi - même si elle est légèrement inférieure à la moyenne - dans la branche de l'enseignement (63,9\%), qui laisse présager l'insertion de la société espagnole dans la société de la connaissance, et dans celle du secteur traditionnel du commerce, dont la croissance relativement moindre $(48,8 \%)$ est pourtant très importante du point de vue de sa participation à la croissance totale $(12,6 \%)$.

Tableau 1 : Emploi et augmentation de l'emploi par branche productive et distribution par branche de l'augmentation totale (1994-2007)

\begin{tabular}{|c|c|c|c|c|c|}
\hline Branches & $\begin{array}{c}1994 \\
\text { (milliers) }\end{array}$ & $\begin{array}{c}2007 \\
\text { (milliers) }\end{array}$ & $\begin{array}{l}\text { Différences } \\
\text { (VA) }\end{array}$ & $\begin{array}{c}\text { Différences } \\
\text { en \% }\end{array}$ & $\begin{array}{c}\text { Part dans } \\
\text { l'augmentation totale } \\
\end{array}$ \\
\hline Toutes & 12186,1 & 20356,0 & 8169,9 & 67,0 & 100 \\
\hline Industries Manufacturières & 2417,2 & 3089,8 & 672,6 & 27,8 & 8,2 \\
\hline Bâtiment & 1119,0 & 2697,3 & 1578,3 & 141,0 & 19,3 \\
\hline Commerce, réparation véhs. & 2102,3 & 3128,6 & 1026,3 & 48,8 & 12,6 \\
\hline Hôtellerie & 741,3 & 1450,5 & 709,2 & 95,7 & 8,7 \\
\hline Activités immobilières, services aux entreprises & 629,4 & 2017,1 & 1387,7 & 220,5 & 17,0 \\
\hline Enseignement & 678,8 & 1112,3 & 433,0 & 63,9 & 5,3 \\
\hline Activités sociales, services personnels & 428,8 & 846,2 & 417,4 & 97,3 & 5,1 \\
\hline Foyers avec employés domestiques & 331,7 & 770,0 & 438,3 & 132,1 & 5,4 \\
\hline
\end{tabular}

Source: Institut national de statistique espagnol et traitement propre.

Note: On n'a retenu que les branches ayant des variations statistiques qui peuvent être considérées significatives. Soit les branches dont l'emploi a augmenté au-dessus de l'augmentation globale, soit celles qui ont eu une participation à l'augmentation de plus de $10 \%$ (le nombre de branches traitées par l'Institut national de la statistique dans le tableau original est de 17). La dernière colonne ne somme pas 100 parce qu'on ne retient pas toutes les branches.

Du point de vue de la qualité des emplois créés, trois points forts doivent être soulignés. Premièrement, même si on constate une forte croissance à tous les niveaux de qualification, la croissance des niveaux inférieurs est de beaucoup la plus élevée; «la croissance des emplois à compétences peu élevées, écrit Immaculada Cebrián, a connu une hausse de 70,4\%, et celle des emplois à compétence élevées de 55,1\%». Deuxièmement, tous les experts notent une très forte persistance de l'emploi temporaire (1). Pour beaucoup d'auteurs, c'est l'aspect qui caractérise le mieux l'emploi espagnol. Ce taux se situe entre 30 et $35 \%$ des emplois salariés, ce qui est très au-dessus de celui de l'Union européenne à quinze où il ne dépasse pas $16 \%$. Il a de plus un caractère de permanence - il n’a pas pratiquement changé depuis le début des années 1990 - et résiste à toutes les mesures de politique de l'emploi orientées vers sa réduction. Les syndicats espagnols en ont fait leur principal cheval

(1) L'emploi temporaire en Espagne englobe diverses formes de contrats à durée limitée ou d'interim. Voir Migélez et Prieto dans ce numéro. 
de bataille, l'identifiant au problème de la précarité. L'emploi temporaire est devenu en Espagne un phénomène social à forte dimension politique. Fausto Miguélez et Carlos Prieto essaient d'en dégager les traits principaux et sa spécificité par rapport à la situation des autres pays de l’Union européenne. Le troisième aspect touche à l'évolution négative des salaires perçus. D'après l'Employment Outlook 2007 (Perspectives de l'emploi, OCDE, 2007), entre 1995 et 2005, le salaire moyen réel en Espagne diminue de $4 \%$, évolution qui, en cohérence avec les deux aspects précédents, s’explique par l'augmentation du poids relatif des emplois à bas salaires considérés très peu productifs. Ainsi, à côté d'une croissance de l'emploi extrêmement positive, on constate des aspects qui le sont moins, comme le poids très supérieur à la moyenne de la croissance des emplois non qualifiés et des emplois à bas salaires, et l'existence persistante d'un taux de travail temporaire particulièrement élevé.

L'analyse de cette évolution de l'emploi (côté offre) doit être complétée par celle des caractéristiques de la population concernée (côté demande) et par l'analyse des conditions de «protection sociale» dans laquelle cette évolution a eu lieu. L'évolution de l'emploi tel qu'on vient de la décrire n’aurait pas été possible sans l'incorporation au marché du travail espagnol de trois catégories nouvelles de travailleurs: les immigrés extra-communautaires, les femmes et les jeunes. Ces catégories sont «nouvelles» par rapport au marché de travail de cette «décennie surprenante», mais leur caractère de nouveauté est différent dans chaque cas. Les immigrés (extra-communautaires) sont «nouveaux» dans le sens le plus radical: avant, ils n'étaient pas là - ou quasiment pas (le graphique élaboré par Lorenzo Cachón sur l'évolution des cartes de séjour accordées montre que le grand saut en avant n’a lieu qu'à partir de 1996); aujourd'hui, ils sont, d'après le Boletín Estadístico de Extranjeria e Inmigración du ministère du Travail espagnol, près de 4 millions à détenir un certificat de registre (ce qui représente $10 \%$ de la population totale) et un peu plus de 2 millions à avoir un emploi (soit environ $10 \%$ de la population occupée). Les femmes avaient commencé leur entrée sur le marché de l'emploi dès la moitié des années 1980, mais leur taux d'activité ne sera comparable à celui de femmes de l’Union européenne à quinze que dans les années de la «décennie »; l'évolution du taux d'activité parmi les femmes en âge de travailler est la suivante: 1987: 42,9\%; 1995: 46,9\%; 2005 : $56,3 \%$. Les jeunes sont "nouveaux» parce que, même s’ils ont été toujours présents sur le marché du travail espagnol, la jeunesse de la fin du siècle a très peu de choses en commun avec celle des années 1970 et 1980. Dans les trois cas, la «nouveauté» implique un rapport à l'emploi qui est, en termes généraux, différent de celui des travailleurs adultes natifs.

On vient de le voir, l'incorporation massive des femmes à l'emploi débute dans la seconde moitié des années 1980, mais la consolidation de cette tendance n’a lieu que dans les années suivantes. Teresa Torns, dans son article sur l'emploi des femmes, rappelle que ce qui est le plus significatif dans ce changement, c'est qu'il atteint surtout les femmes dans les âges centraux de la vie, et plus significativement encore, les femmes mariées en âge d'avoir des enfants en bas âge; entre 1985 et 2005 les taux d'activité de femmes mariés entre 35 et 39 ans se sont plus que décuplés (voir tableau 2).

Tableau 2: Taux d’activité des femmes mariées entre 35 et 39 ans (1985-2005)

\begin{tabular}{|r|c|c|c|c|}
\hline Âge année & $\mathbf{1 9 8 5}$ & $\mathbf{1 9 9 5}$ & $\mathbf{2 0 0 0}$ & $\mathbf{2 0 0 5}$ \\
\hline $35-39$ & 27,8 & 54,3 & 61,0 & 63,6 \\
\hline
\end{tabular}

Source: enquête sur la population active (EPA) et traitement propre.

La situation de l'emploi des femmes s'est ainsi beaucoup améliorée en Espagne pendant les dernières années et s'est rapprochée de celle de l’Union européenne à quinze. Mais, écrit Teresa Torns, les différences persistent. Non seulement parce que leur taux d'activité est encore inférieur, mais surtout parce que l'emploi créé est de très basse qualité. La différence par rapport à l'emploi des hommes est nette: toutes les conditions négatives d'emploi touchent les femmes avec plus d'intensité (voir tableau 3).

Tableau 3 : Distribution par sexe des situations d'emploi socialement significatives (2005)

\begin{tabular}{|r|r|r|r|r|r|r|r|r|r|}
\hline \multicolumn{2}{|c|}{ Taux d'activité } & \multicolumn{2}{c|}{ Taux de chômage } & \multicolumn{2}{c|}{ Taux de CDD } & \multicolumn{2}{c|}{$\begin{array}{c}\text { Taux d'emplois } \\
\text { à temps partiel }\end{array}$} & \multicolumn{2}{c|}{$\begin{array}{c}\text { Salaires mensuels en } \\
\text { indice (hommes = 100) }\end{array}$} \\
\hline Hommes & Femmes & Hommes & Femmes & Hommes & Femmes & Hommes & Femmes & Hommes & \multicolumn{1}{c|}{ Femmes } \\
\hline 82,2 & 59,3 & 7,3 & 12,2 & 31,6 & 35,5 & 4,2 & 24,8 & 100 & 69,9 \\
\hline
\end{tabular}

Source: Institut national de statistique espagnol et traitement propre.

Lecture: taux d'activité: population entre 16 et 64 ans. Taux de CDD:\% sur la totalité de salariés de chaque groupe. Taux d'emplois à temps partiel:\% sur l'ensemble de salariés de chaque groupe. Salaires mensuels: d'après les données de 2006 de l'Agence nationale d'impôts. 